\title{
5. Les constructions verbales en co(n)texte et en contraste : le cas du verbe observer
}

Dominique Willems

Universiteit Gent

\section{Les verbes de perception visuelle en français : organisation syntaxique et lexicale}

Le champ sémantique des verbes de perception visuelle s'organise traditionnellement autour des deux verbes principaux : voir et regarder. Ces verbes partagent d'une part les propriétés syntaxiques et sémantiques essentielles des verbes de perception, à savoir la possibilité d'entrer dans une structure infinitive directe (ex. 2) et une structure à relative attributive (ex. 3), à côté de la structure transitive simple (ex. I) :

(I) a. Dès qu'ils voient un uniforme, ils pètent les boulons. ${ }^{\mathrm{I}}$

b. Noreddine regarde le ciel en espérant qu'il va neiger ou faire très froid.

(2) a. C'est plaisir de voir tous les petits bourgeons poindre à fleur d'écorce.

b. Elle restait des heures à regarder les grands danser paso-doble, valses et autres tangos.

(3) a. "C'est à la fois dégoûtant parce qu'on voit ses ongles acérés qui en-trent dans la chair, et libérateur parce que cette dame ose poser nue », commente une grande blonde à lunettes avant de grimper dans un wagon.

b. De retour du travail, Oscar et Karl, des ingénieurs, prennent le temps de regarder les images qui s'étalent sur de vastes panneaux.

Cette famille de trois constructions est en effet spécifique pour l'ensemble des verbes de perception en français (cf. Willems I981, I983; Willems \& Defrancq 2000) et peut être corrélée à un sémantisme

Comment citer ce chapitre :

Willems, Dominique, Les constructions verbales en co(n)texte et en contraste : le cas du verbe observer. In: Engwall, Gunnel \& Fant, Lars (eds.) Festival Romanistica. Contribuciones lingüísticas - Contributions linguistiques - Contributi linguistici Contribuições linguísticas. Stockholm Studies in Romance Languages. Stockholm: Stockholm University Press. 201 5, pp. 92-I09. DOI: http://dx.doi.org/IO.I6993/bac.e. License: CC-BY 
précis : la possibilité pour un sujet (généralement humain) de percevoir simultanément un objet et un procès lié à cet objet.

Tant voir que regarder admettent par ailleurs une construction à attribut de l'objet, véhiculant un sens de jugement (ex. 4) (cf. Willems \& Defrancq 2000) :

(4) a. Elle est interne en première STT au lycée Simone-Weil de Dijon " du nom de la résistante ", précise-t-elle, assez contente de ce qu'elle a décidé de voir comme une coïncidence.

b. Ces phénomènes, qui exigent une grande mobilité et une " adaptabilité » des travailleurs, peuvent être regardés comme positifs s'ils concourent à améliorer la compétitivité de l'économie.

Les deux verbes s'opposent toutefois sur bien des points. Sur le plan syntaxique, c'est surtout le comportement par rapport à la construction complétive qui différencie les deux verbes, regarder n'admettant pas cette structure contrairement à voir (ex. 5, 6). Regarder se construit par ailleurs fréquemment avec un complément prépositionnel locatif (ex. 7), propriété que le verbe partage avec les verbes de mouvement directionnels.

(5) Regardez à votre gauche et à votre droite, et vous verrez ("regarderez) que la défense des droits de l'homme implique des sacrifices.

(6) Je suis surpris de voir ("regarder) que les hommes sont là, tranquillement accroupis dans la vase, et je comprends que les Boches sont en train de repousser une attaque imaginaire.

(7) a. Souvent, entre deux visites, il regarde ("voit) par terre.

b. Le tableau doit-il être regardé de bas en haut ou de gauche à droite?

Ces différences syntaxiques s'accompagnent de différences sémantiques importantes : alors que voir est un verbe d'état exprimant pour son sujet 'expérienceur' une perception réussie, regarder exprime une activité perceptive intentionnelle et dirigée, pas nécessairement réussie, de la part d'un agent actif et volontaire. Voir, par le biais de la construction complétive passe du domaine perceptif physique au domaine cognitif, en passant par la perception indirecte ${ }^{2}$. L'analyse des éléments contextuels (en particulier les adverbes de manière) accompagnant ces verbes, révèle l'importance du sujet agentif dans le cas de regarder, celle de l'objet ou du prédicat dans le cas de voir :

(8) a. Comme chaque matin, il regarde avec un rien de tendresse le cimetière qui s'étend devant son immeuble délabré.

b. L'homme que l'on voyait de dos depuis le début de l'émission se re-tourne enfin : c'est Bertrand Delanoë, sénateur socialiste de Paris. 
Nous résumons les propriétés syntaxiques et sémantiques dans les Tableaux I et 2 ci-dessous, en marquant en gras les structures spécifiquement perceptives :

Tableau 1. voir /v/ regarder : différences syntaxico-sémantiques

\begin{tabular}{llcc}
\hline structures & sens structural & voir & regarder \\
\hline $\mathrm{SNI}+\mathrm{V}+\mathrm{SN}_{2}$ & (perception) & + & + \\
$\mathrm{SNI}+\mathrm{V}+\mathrm{SN}_{2}+\mathrm{INF}$ & perception directe & + & + \\
$\mathrm{SNI}+\mathrm{V}+\mathrm{SN} 2+$ rel. attrib. & perception directe & + & + \\
$\mathrm{SNI}+\mathrm{V}+$ que $+\mathrm{P}_{\mathrm{IND}}$ & perception (directe et & + & - \\
& indirecte) /cognition & & + \\
$\mathrm{SNI}+\mathrm{V}+\mathrm{SNprép}_{\mathrm{LOC}}$ & perception directe + & - & + \\
& mouvement directionnel & & \\
$\mathrm{SN}$ & jugement & + & + \\
\hline
\end{tabular}

Tableau 2. voir /v/ regarder : différences sémantico-lexicales

\begin{tabular}{ll}
\hline \multicolumn{1}{c}{ voir } & \multicolumn{1}{c}{ regarder } \\
\hline $\begin{array}{l}\text { généralement non agentif } \\
\text { perception réussie } \\
\text { perception directe et indirecte } \\
(\rightarrow \text { cognition })\end{array}$ & $\begin{array}{l}\text { agentif, volontaire, intentionnel } \\
\text { résultat perceptif non garanti } \\
\text { perception directe }\end{array}$ \\
état/achèvement & activité + mouvement \\
orienté vers l'objet & orienté vers le sujet \\
\hline
\end{tabular}

La presque totalité du lexique verbal de perception visuelle se laisse classer dans une des deux sous-classes (cf. Tableau 3):

Tableau 3. Le lexique de la perception visuelle

Percevoir par les yeux :

\begin{tabular}{ll}
\hline A) voir & B) regarder \\
apercevoir, entrevoir, revoir, & admirer, contempler, examiner, scruter, \\
découvrir, déceler, discerner, & inspecter, guetter, épier, fixer, guigner, \\
distinguer, remarquer, repérer, & lorgner, loucher, zieuter, bigler, \\
surprendre, imaginer, envisager (...) & dévisager, toiser, reluquer (...) \\
\hline
\end{tabular}




\section{La place particulière du verbe observer dans le champ de la perception visuelle}

Le verbe observer occupe toutefois une place particulière dans le champ : il présente des caractéristiques à la fois de voir et de regarder : avec ce dernier il partage le caractère agentif et volontaire du sujet, avec le premier l'aspect réussi de la perception ainsi que la possibilité de se construire avec une complétive et d'adopter de ce fait les caractéristiques plus cognitives de voir. Nous reproduisons ci-dessous le Tableau 2 en marquant en caractères gras les propriétés sémanticolexicales du verbe observer:

Tableau 4. observer : propriétés sémantico-lexicales par rapport à voir et regarder

\begin{tabular}{ll}
\hline voir & regarder \\
\hline généralement non agentif & agentif, volontaire, intentionnel \\
perception réussie & résultat perceptif non garanti \\
perception directe et indirecte $(\rightarrow$ cognition $)$ & perception directe \\
état/achèvement & activité + mouvement \\
orienté vers l'objet & orienté vers le sujet \\
\hline
\end{tabular}

Nous examinerons plus en détail les propriétés syntaxiques, sémantiques et lexicales du verbe afin de mieux cerner sa position particulière dans le champ de la perception. Notre étude est basée sur une analyse d'un ensemble de 600 exemples, pris d'une part à un corpus journalistique (le Monde 2006 : I 50 exemples, Libération 2006 : I 50 exemples), au corpus littéraire de Frantext d'autre part (300 exemples).

\subsection{Observer : sens et structures}

Le verbe présente deux sens principaux, relevés par l'ensemble des dictionnaires, sans lien apparent entre eux : un premier sens "perceptivo/ cognitif ", de loin le mieux représenté dans le corpus (ex. 9), et un deuxième sens, historiquement le premier, non perceptif, que nous appellerons "d'observance »3 (ex. Io), et que nous ne retrouvons que dans la structure transitive nominale, avec un nombre restreint et bien délimité de $\mathrm{SN}_{2}$ (la loi, des réserves, la grève, une minute de silence etc.).

(9) Pas très loquace, il observait les gens, les choses autour de lui, avec une attention soutenue. (Alphonse Boudard, 1995, Frantext) 
(Io) Par le silence qu'il observe habituellement, refusant radicalement les interviews depuis des années, le romancier sud-africain John Maxwell Coetzee s'attire un succès mêlé de crainte, les rares fois où il consent à se montrer en public. (Le Monde, 29.9.2006)

Le sens «perceptivo/cognitif » présente quant à lui quatre sousensembles de constructions, liés à des sous-sens spécifiques :

(a) Dans son sens perceptif pur (physique, direct ou indirect) le verbe utilise le plus souvent une structure transitive nominale avec un objet de nature essentiellement concrète (ex. II $)^{4}$. On relève également les autres structures typiquement perceptives, telle la structure à infinitif (ex. I2) ou la relative attributive (ex. I3), ainsi que quelques interrogatives indirectes en comment (ex. I4) :

(I I) Le visiteur observe furtivement le paquet de cigarettes, le cendrier posés sur le bureau de Beaune. (Jean-Bertrand Pontalis, Frantext, I996)

(I2) Mais c'est avec des rires incrédules qu'ils observent finalement le vendeur cracher dans un grand récipient en inox au terme de la démonstration. (Libération, I997)

(I3) Non loin, trois jeunes originaires de Pärnu, dans le sud-ouest de l'Estonie, observent des grands-mères qui se recueillent devant le soldat de bronze. (Libération, I997)

(I4) Il sera intéressant d'observer comment Ségolène Royal va gérer ce phénomène. (Le Monde, 2006)

Dans cet emploi, observer est proche du verbe regarder (perception active, intentionnelle), tout en présentant des spécificités lexicales que nous développerons sous 2.2. C'est le sens le plus courant d'observer dans les deux corpus distingués.

(b) Dans le sens plus cognitif de 'constater', le verbe s'emploie essentiellement dans la structure complétive (suivi d'une que P) (ex. I 5) ou avec un $\mathrm{SN}_{2}$ de nature abstraite (tendance, mouvement, évolution, hausse etc.) (ex. I6). Le sème de perception reste présent, mais il s'y ajoute une dimension interprétative et déductive. C'est en quelque sorte le résultat de la perception qui est présenté comme objet du verbe et non la perception proprement dite.

(I 5) J'observe qu'un autre sujet revient souvent dans les débats concernant la Turquie : la question arménienne. (Le Monde, 2006)

(16) C'est pourquoi on observe un retour progressif vers des supports plus risqués. Ce constat a incité de nombreux établissements financiers à repenser leur offre. (Le Monde, 2006) 
(c) Le verbe peut finalement s'employer avec un sens causatif, de perception ('faire voir') (ex. I7), ou de cognition ('faire savoir, dire') (ex.I 8), soit en explicitant le sème causatif par l'utilisation de l'opérateur faire, soit, plus rarement, en utilisant simplement la structure bitransitive à complément nominal indirect (à $+\mathrm{SN}_{\text {humain }}$ ). C'est surtout le sens de 'dire' qui s'est développé, en particulier dans l'incise (ex. I9). Ce sens est plus fréquent dans le corpus de presse que dans le corpus littéraire (cf. Tableau 5).

(I7) Lorsque leur ignorance des réalités atteignait à la candeur, c'est moi qui les mettais en garde, leur faisais observer toutes les pommes pourries, ou tavelées, pour ne rien dire des patates, adroitement dissimulées parmi celles qui l'étaient un peu moins. (Jean Rolin, I996, Frantext)

(I 8) Patrick fit observer qu'il ne risquait pas grand-chose, mais la maîtresse dit que non, c'était une question de principe. (Emmanuel Carrère, I995, Frantext)

(19) "Il existe au sein des populations néandertaliennes des tendances évolutives ", observe-t-elle. (Libération, I997)

Le tableau 5 ci-dessous synthétise les divers emplois et leur fréquence dans le corpus. Les deux corpus journalistiques ne présentant pas de différences significatives, nous les avons regroupés. Nous présentons en gras et soulignés les chiffres affichant des différences notables:

Tableau 5. Observer : structures et sens

\begin{tabular}{|c|c|c|c|}
\hline \multirow[t]{2}{*}{ Sens \& structure } & \multicolumn{2}{|c|}{ Texte } & \multirow[t]{2}{*}{ Total } \\
\hline & Frantext & Journaux & \\
\hline \multicolumn{4}{|l|}{ I. sens perceptif (394 ex) } \\
\hline - $\mathrm{SNI}+\mathrm{V}+\left[\mathrm{SN}_{2}{ }_{\text {concret+ abstr }}\right]$ & 222 & $\mathrm{I} 45$ & 367 \\
\hline - $\mathrm{SN}_{\mathrm{I}}+\mathrm{V}+\mathrm{SN}_{2}+\mathrm{inf} / \mathrm{rel} / \mathrm{ppr}$ & 8 & 7 & I5 \\
\hline - $\mathrm{SN}_{\mathrm{I}}+\mathrm{V}+\mathrm{QI}$ & 3 & 4 & 7 \\
\hline - $\mathrm{SNI}+\mathrm{V}+q u e \mathrm{P}$ & 2 & 3 & 5 \\
\hline \multicolumn{4}{|l|}{$\begin{array}{l}\text { 2. sens (perceptivo)/cognitif } \\
(« \text { constater } »)(87 \mathrm{ex})\end{array}$} \\
\hline - $\mathrm{SN}_{\mathrm{I}}+\mathrm{V}+\mathrm{SN}_{2}{ }_{\text {abstr }}$ & I4 & 26 & 40 \\
\hline - $\mathrm{SN} I+\mathrm{V}+q u e \mathrm{P}$ & $\overline{24}$ & $\overline{23}$ & 47 \\
\hline \multicolumn{4}{|l|}{$\begin{array}{l}\text { 3. sens causatif de perception } \\
\text { («faire voir ») (5 ex) }\end{array}$} \\
\hline - $\mathrm{SN}_{\mathrm{I}}+\mathrm{V}+\left[\mathrm{a}+\mathrm{SN}_{2}\right]+q u e \mathrm{P}$ & - & 2 & 2 \\
\hline - $\mathrm{SN}_{\mathrm{I}}+$ faire $\mathrm{V}+\left[\mathrm{a}+\mathrm{SN}_{2}\right]+q u e \mathrm{P}$ & I & 2 & 3 \\
\hline
\end{tabular}




\begin{tabular}{|c|c|c|c|}
\hline \multirow[t]{2}{*}{ Sens $\&$ structure } & \multicolumn{2}{|c|}{ Texte } & \multirow[t]{2}{*}{ Total } \\
\hline & Frantext & Journaux & \\
\hline \multicolumn{4}{|l|}{$\begin{array}{l}\text { 4. sens causatif de cognition } \\
\text { (« faire savoir, dire ») }(87 \mathrm{ex})\end{array}$} \\
\hline - $\mathrm{SNI}+\mathrm{V}$ ( en incise) & $\underline{6}$ & $\underline{62}$ & 68 \\
\hline - $\mathrm{SN}_{\mathrm{I}}+\mathrm{V}+\left[\mathrm{a}+\mathrm{SN}_{2}\right]+q u e \mathrm{P}$ & 2 & 4 & 6 \\
\hline - $\mathrm{SN}_{\mathrm{I}}+$ faire $\mathrm{V}+\left[\grave{\mathrm{a}}+\mathrm{SN}_{2}\right]+$ que $\mathrm{P}$ & $\underline{13}$ & - & 13 \\
\hline \multicolumn{4}{|l|}{$\begin{array}{l}\text { 5. sens d'observance ( « se conformer à, } \\
\text { suivre ») }(26 \mathrm{ex})\end{array}$} \\
\hline - $\mathrm{SN}_{1}+\mathrm{V}+\mathrm{SN}_{2}$ & $\underline{5}$ & $\underline{22}$ & 27 \\
\hline Total & 300 & 300 & 600 \\
\hline
\end{tabular}

\subsection{Observer dans son sens perceptif : analyse co(n)textuelle détaillée}

Une analyse détaillée des contextes d'apparition du verbe, en particulier la fréquence et la nature des compléments adverbiaux, la nature sémantique des sujets et des objets, ainsi que les combinatoires lexicales de verbes, révèle des traits sémantiques spécifiques à la perception rapportée par observer. Nous donnons pour chaque trait les indices contextuels et quelques exemples révélateurs :

(a) l'intensité de la perception : la présence d'adverbes tels attentivement, soigneusement, avec intérêt, méticuleusement, avec une curiosité particulière, sous toutes les coutures (...), ainsi que les combinaisons fréquentes avec des verbes tels ausculter, inspecter, examiner, étudier (...) sont révélateurs du caractère minutieux et non banal de la perception rapportée par observer. Ce trait va de pair avec le caractère intentionnel du procès ( $j e$ m'appliquais à observer, je m'exerce à observer, je me réveillais pour observer etc.) et le trait 'intéressant' de l'objet observé (cf. infra). La présence fréquente de l'instrument d'observation (à la jumelle, à la loupe, via des caméras, à l'œil nu...) renforce l'intensité de la perception. On précise aussi régulièrement le point de vue de l'observateur (de haut, de loin, d'en bas, à travers la vitre, derrière la fenêtre, de la cuisine, à la dérobée, furtivement, du coin de l'œil, de plus près...).

(20) La Suède est observée avec intérêt, et souvent enviée, pour avoir réussi à « réinventer » le modèle social-démocrate. (Le Monde 2006) 
(2I) Pas très loquace, il observait les gens, les choses autour de lui, avec une attention soutenue. (Alphonse Boudard, I995, Frantext)

(22) Comme il ne comprenait rien, il les a observées à la loupe. (Valérie Mréjen, 200I, Frantext)

L'intensité de l'observation peut être telle qu'elle affecte la personne observée, qui 'se sent' ou 'se sait' observée :

(23) Au bout d'un instant, il changea d'attitude, allongea une grande main vers le sac (...). Peut-être se savait-il observé. (Julien Green, I950, Frantext)

(b) l'aspect duratif et cumulatif du procès : l'intensité de la perception va de pair avec une certaine durée (longuement, longtemps, plusieurs semaines, nous restions des heures à observer, passer ses journées (deux mois) à observer etc.). Elle présente par ailleurs souvent un aspect cumulatif dans le temps et dans l'espace (depuis huit jours, depuis une décennie, depuis trente ans, d'année en année, comme chaque soir, depuis vingt sondages; partout en Europe et aux EtatsUnis, en Italie, au Mexique en Allemagne etc.). Par un raccourci logique, le résultat synthétique de la perception est souvent présenté en tant que COD du verbe (la hausse, la tendance, la progression etc.), ajoutant au sens perceptif une dimension clairement interprétative :

(24) Plus tard, dans le silence du soir, j'observais longuement la plaine déserte avec les rayures sombres de l'herbe arrachée çà et là. (Andreï Makine, I995, Frantext)

(25) "C'est une tendance que l'on observe partout en Europe et aux EtatsUnis ", reconnaît Arlette Chabot. (Le Monde 2006)

(26) Depuis une décennie, d'année en année, on observe une augmentation moyenne des prix de $3 \%$ ! (Le Monde 2006)

(c) la télicité du procès et l'aspect déductif de la perception : ce qui frappe par ailleurs c'est le caractère télique de l'action d'observer: on observe pour analyser, étudier et finalement connaître. L'observation a un but qui se situe clairement au-delà de la perception (cf. Willems 2000 : I 8I). C'est généralement la connaissance qui est visée (pour savoir, pour conclure, pour voir, pour discerner), ou, plus modestement, la constatation par déduction synthétique (constater, repérer, déceler) ou, dans le corpus littéraire, la description ou l'inspiration (pour décrire, pour peindre, pour s'en inspirer). Si l'aspect télique est souvent explicitement marqué par la préposition pour, il ressort aussi de la simple 
succession des verbes (observer et voir, observer et constater, observer et supposer, observer et tirer la conclusion) :

(27) Je procède comme suit : j'observe attentivement le ventre de l'animal pour y repérer les parasites. (Lydie Salvayre, I995, Frantext)

(28) Le dispositif permet d'observer les évolutions du monde du travail, des relations sociales et du rapport à l'outil en tentant de comprendre ce que l'on transmet, comme de déterrer ce que l'on enfouit. (Le Monde 2006)

(29) [...] et il fallait l'observer avec beaucoup d'attention pour voir la grâce d'une longue paupière à la Garbo voilant à demi un œil très vif. (Annie Duperey, I999, Frantext)

(d) Les aspects intentionnel, intensif et télique du procès soulignent par ailleurs l'importance de l'objet, qui dans l'observation importe bien plus que l'agent. Ceci se traduit entre autres par la fréquence de la structure passive ou relative, mettant l'objet en position focale et permettant d'omettre aisément le sujet (cf. Willems, 2000). Ce dernier est souvent aussi indéfini ou général (fréquence du pronom on).

(30) Tu l'intéresses, comprends-tu ? Il t'a vu l'autre jour, il t'a observé [...] lui et moi, nous avons passé la soirée entière à parler de toi. (Julien Green, I950, Frantext)

(3I) A observer tout ce qui oppose désormais Ségolène Royal et François Hollande, on comprend qu'il y avait bien urgence à ce que le couple officialise sa séparation. (Le Monde 2006)

(32) L'essor des pentecôtistes s'observe dans toute l'Amérique latine - où vit près d'un catholique sur deux - et explique pourquoi Benoît XVI a choisi de se rendre dans cette région stratégique pour l'avenir de l'Eglise. (Le Monde 2006)

Ces diverses caractéristiques font d'observer le verbe par excellence pour désigner l'activité scientifique empirique, où, dans un souci d'objectivité, le sujet est en quelque sorte neutralisé. Elles expliquent également la fréquence élevée du verbe dans des contextes littéraires, l'observation d'un sujet (souvent humain) menant dans ce cas à des interprétations de nature plus psychologique.

L'analyse détaillée sur le plan lexical nous permet de proposer pour le verbe observer la définition suivante : regarder attentivement et de façon répétée un objet intéressant, afin de l'analyser, de faire des déductions et finalement de le connaître. 
Sur un plan contextuel plus large, observer apparaît de façon privilégiée dans les cadres sémantiques ${ }^{5}$ suivants :

(a) la recherche scientifique (l'observation constituant une des étapes essentielles de la recherche empirique)

(33) Mais nous n'avons pas pour le moment observé de Culicoides imicola le principal vecteur de la FCO en Europe du Sud - dans nos prélèvements, et ceux de nos collègues européens ne l'ont pas non plus révélé. (Le Monde 2006)

(34) Un archisémème peut ne correspondre à aucun " mot " par exemple pour la série échelle, escalier, etc. On peut quelquefois observer la création d'archisémèmes, par exemple pour la série professeur, instituteur, etc. ou agrume.

(b) l'analyse psychologique (l'objet = comportement humain)

(35) Elle avait eu de nombreuses occasions de l'observer quand, aux vacances d'automne, il chevauchait en voisin jusqu'au château ; rien à faire : elle ne l'aimait pas. (Francoise Chandernagor, I995, Frantext)

(36) Et Charlotte, en observant les gestes quotidiens de sa mère, se disait souvent en souriant : "Mais c'est une vraie Sibérienne ! (Andreï Makine, I995, Frantext)

(c) l'observation des phénomènes de la nature (ciel, étoiles...)

(37) J'observe les constellations - cette fois-ci du chemin (comme hier), et non de la verrière (comme au début). (Raymond Queneau, I928I932, Frantext)

(38) Je venais d'observer longuement un faucon crécerelle - un oiseau auquel s'attache pour moi une signification symbolique particulière perché au sommet d'un tronc. (Jean Rolin, I996, Frantext)

(d) le domaine politique et diplomatique (l'observation impliquant distance et non engagement ; cf. le dérivé nominal observateur)

(39) Sa prudence et sa circonspection sont partagées. "Pour l'instant on observe ", résume le président de la CFTC, Jacques Voisin. (Le Monde 2006)

(40) Alors que certains responsables du secteur humanitaire du Hezbollah observent avec une méfiance ostensible la visite des casques bleus français à l'hôtel Dana. (Le Monde 2006) 


\subsection{Observer : polysémie 'logique' et ambiguïté}

Le sémantisme particulier du verbe permet de rendre compte de sa polysémie. L'objet de l'observation présentant un intérêt particulier et les résultats donnant lieu à des déductions intéressantes, il est normal de vouloir les partager (ex. 4I). Cette dimension intersubjective, qu'on relève également pour le verbe remarquer, explique la fréquence de l'emploi causatif, que ce soit un causatif de perception ('montrer') (ex. 42 ) ou plus fréquemment un causatif de cognition ('faire savoir/ dire') (ex. 43):

(4I) Ligne claire et ferme du cinéaste qui, sans commentaire, observe et fait voir. (Le Monde, 2006)

(42) [...] c'est moi qui les mettais en garde, leur faisais observer toutes les pommes pourries, ou tavelées, pour ne rien dire des patates, adroitement dissimulées parmi celles qui l'étaient un peu moins. (Jean Rolin, I996. Frantext)

(43) [...] mais Marie m'a fait observer (constatant par ailleurs la détérioration évidente de mon humeur), que c'était plutôt moi (entre autres) qui était puni par cet acte politiquement courageux, certes. (Jacques Roubaud, I997, Frantext)

Avec le sens de 'dire', le verbe s'approprie sans problème la structure en incise, propre aux paroles rapportées.

(44) «En dépit de la volonté des deux entreprises de la minimiser, la communication des griefs est extrêmement sévère, observe un expert bruxellois, si rien n'est fait, il n'est pas exclu que la fusion soit interdite. » (Le Monde 2006)

Le lien avec l'emploi performatif d'observance ('se conformer à, suivre') est plus difficile à expliquer : observer rejoint dans ce cas un verbe comme écouter, qui, en dehors de son sens purement perceptif peut également prendre la valeur performative d' " exaucer ». Les traits intentionnel et intensif forment sans doute le lien entre ces deux valeurs à première vue fort différentes.

La polysémie inhérente du verbe ne donne que rarement lieu à de réelles ambiguïés : les structures syntaxiques partiellement différentes et la nature lexicale de l'objet rendent l'interprétation le plus souvent transparente. Il reste toutefois quelques cas intéressants d'ambiguïté réelle, comme en témoignent les exemples ci-dessous : entre le sens perceptif et performatif (ex. 45, 46), entre le sens perceptif et le dire (ex. 47) : 
(45) D'emblée quelque chose cloche dans l'ordre du rituel qu'observent la femme en noir et ses trois fils. (Libération, I997) ['regarder' ou 'suivre']

(46) Train de nuit observe les limites entre le politique (entendre : le domaine de la répression) et l'intime (la liberté des yeux pour pleurer) : ici même, la surveillance permanente qui menace les couples adultères. (Libération, I997) ['regarder' ou 'se conformer à']

(47) Je me souviens d'avoir observé que nous étions en nombre pair et, excepté Orazio et moi, tous semblables par leur robustesse et leur appartenance à une classe intermédiaire. (Hector Bianciotti, I995, Frantext) ['constater' ou 'dire']

Un co(n)texte plus large ou une connaissance extra-linguistique plus approfondie suffit bien souvent à désambiguïser le verbe.

Dans la Figure I, nous visualisons la configuration polysémique du verbe, en précisant les structures propres à chaque sens et en marquant la fréquence d'usage des différents emplois.
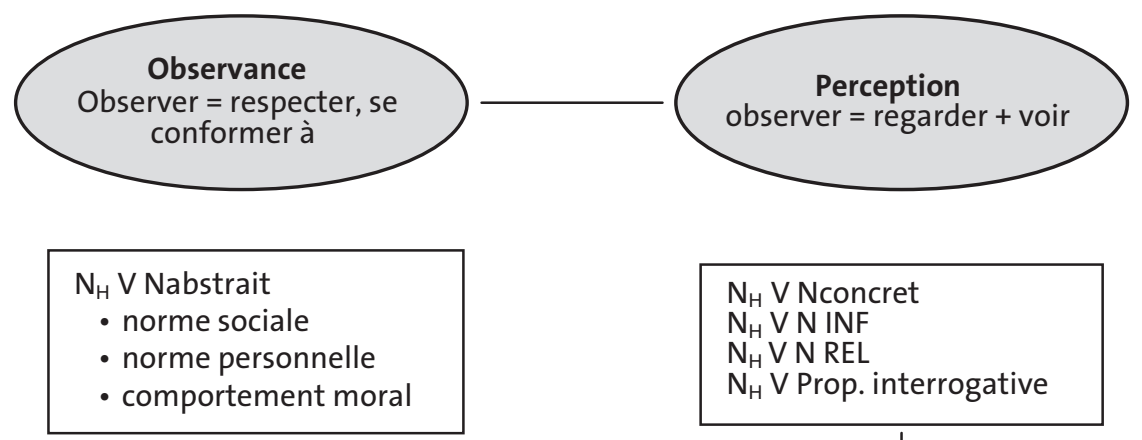

$\mathrm{N}_{\mathrm{H}}$ V Nabstrait

- norme sociale

- norme personnelle

$\mathrm{N}_{\mathrm{H}} \mathrm{V}$ Nconcret

$\mathrm{N}_{\mathrm{H}} \mathrm{V} \mathrm{N}$ INF

$\mathrm{N}_{\mathrm{H}}$ V N REL

$\mathrm{N}_{\mathrm{H}} \mathrm{V}$ Prop. interrogative

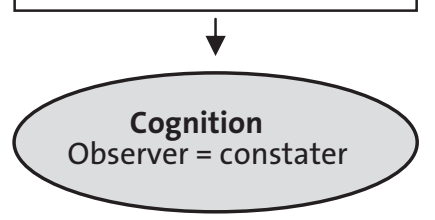

NH V Nabstrait

NH V que PROPIND

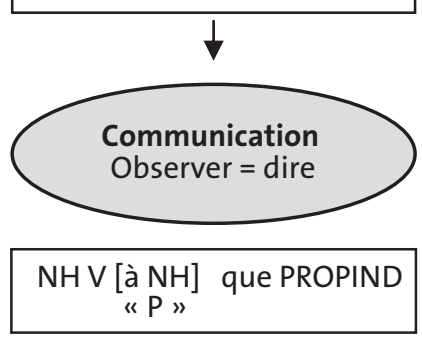

Figure 1. La polysémie d'observer 


\section{Observer en contraste}

\subsection{Observer $/ v /$ regarder et voir}

$\mathrm{Si}$, sur bien des plans, observer se rapproche de regarder (caractère agentif, volontaire et intentionnel du procès), il apparaît comme sémantiquement plus marqué, comme en témoignent les exemples suivants, où les deux verbes sont combinés :

(48) J'observais cela comme on regarde une pile thermoélectrique. (Catherine Pozzi, 1997, Frantext)

(49) La Comtesse regarda son fils comme un entomologiste observe un insecte d'une espèce inconnue. (Françoise Chandernagor, I995, Frantext)

(50) Et voilà que soudain il la regarde! Et même qu'il l'observe! (Françoise Dorin, I997, Frantext)

(5 I) Vous me regardez avec la passion sérieuse que l'on a pour observer au miroir son propre visage. (Catherine Pozzi, I997, Frantext)

D'autres traits spécifiques expliquent la proximité avec le verbe voir : la perception rapportée par observer est, comme celle de voir, une perception réussie, qui mène logiquement à un constat de faits. Celui-ci se traduit alors formellement par l'utilisation de la construction complétive, la structure cognitive par excellence.

(52) "On observe qu'il ressemble beaucoup à celui des grands singes, souligne Jean-Jacques Jaeger, professeur de paléontologie à l'université de Poitiers. (Le Monde 2006)

Les deux verbes se retrouvent aussi fréquemment en coordination ou en juxtaposition, l'observation menant logiquement au « voir » :

(53) Aussi, ce n'est pas sans quelque inquiétude que les stratèges du parti centriste observent les récentes études d'opinion et voient se dessiner l'hypothèse d'un match mettant aux prises Ségolène Royal et Nicolas Sarkozy. (Le Monde 2006)

(54) Je ne sais plus, et ne vois plus rien, du reste, tant j'ai observé avec des émotions contraires. (Philippe Forest, I997, Frantext)

(55) Elle était un peu folle, les nerfs malades, on l'a mise finalement à l'asile, ou peut-être à l'hôpital, mais elle savait observer, et ce don, elle l'a donné à son fils, elle lui a appris à voir. (Geneviève Brisac, I996, Frantext)

En réalité, le verbe observer semble combiner les sémantismes de regarder et de voir : la perception se déroule en deux mouvements : une perception 
physique minutieuse et cumulative (une façon de regarder), menant à une perception mentale déductive et synthétique (une façon de voir). Dans bien des contextes, les deux étapes se confondent dans une sorte de raccourci, n'exprimant explicitement que la deuxième phase du procès.

\subsection{Observer $/ v /$ contempler}

Pour compléter l'analyse du verbe observer et mettre en lumière sa spécificité, il est utile de le comparer à d'autres verbes du champ, qui lui sont proches. Avec contempler ${ }^{6}$, le verbe présente plusieurs traits lexicaux en commun : les deux verbes expriment une perception active et volontaire (du type regarder), présentant une certaine durée et intensité (ex. 56, 57) et portant sur un objet intéressant $($ ex. 58, 59) :

(56) Il contempla son modèle pendant un moment et marmonna quelque chose d'incompréhensible. (Julien Green, 1950, Frantext)

(57) Je suis resté des heures à contempler ce côté-là et les variations de structure que le déclin du soleil y apportait. (Albert T'Serstevens, I963, Frantext)

(58) Il contemple Corte comme s'il était en présence de quelque être extraordinaire. (Albert Camus, I955, Frantext)

(59) [...] un mur un mur je vous demande enfin pourquoi le contempler un mur n'est pas beau un mur n'est pas grand un mur n'est qu'un mur. (Louis Aragon, I956, Frantext)

Les verbes diffèrent toutefois sur plusieurs points : l'aspect télique est beaucoup moins net pour contempler que pour observer et le but de la perception, si but il y a, n'est pas de nature cognitive (observer pour connaître), mais de nature plus spirituelle ou affective : le sujet est en quelque sorte imprégné par l'objet perçu, qui produit sur lui un effet émotionnel certain (ex. 60, 6I). Le verbe n'admet d'ailleurs pas la structure complétive, propre au domaine de la cognition.

(60) Déjà cependant, Rilke nous dit sa joie de contempler une boîte qui ferme bien. (Gaston Bachelard, 1957, Frantext)

(6I) Or l'icône n'est pas faite pour donner l'impression de la vie réelle, mais pour produire sur ceux qui la contemplent un effet d'ordre moral et religieux. (Louis Bréhier, I950, Frantext)

Alors que la perception rapportée par observer est vérifiable et objective, indépendante du sujet, avec contempler elle est, au contraire, fortement influencée par l'état d'âme du sujet. Ici, c'est la subjectivité qui l'emporte (ex. 62, 63). 
(62) Henri revit cette journée de printemps où il contemplait avec nostalgie les pêcheurs à la ligne. (Simone de Beauvoir, I954, Frantext)

(63) Je m'exaltais, comme aux soirs où, derrière des collines bleues, je contemplais le ciel mouvant; j'étais le paysage et le regard : je n'existais que par moi, et pour moi. (Simone de Beauvoir, I958, Frantext)

La contemplation peut aussi être abstraite, intérieure, et dépasser la pure perception, pour atteindre l'essence même de l'objet contemplé :

(64) Jusqu'ici, l'antiquité s'était confondue pour moi avec la Méditerranée; sur l'acropole, dans le forum, j'avais contemplé sans surprise mon propre passé. (Simone de Beauvoir, I954, Frantext)

Les deux cadres sémantiques les mieux représentés sont le contexte religieux et philosophique d'une part', les phénomènes de la nature (paysages, larges étendues) ou les œuvres d'art d'autre part.

\subsection{Observer / $/ \mathrm{c}$ considérer}

La comparaison d'observer avec considérer ${ }^{8}$ est intéressante elle aussi, tant sur le plan synchronique que diachronique9. Les deux verbes partagent les mêmes structures syntaxiques perceptives et cognitives, auxquelles considérer ajoute encore, tout comme voir et regarder, la structure évaluative avec attribut de l'objet (cf. Tableau 6). Les deux verbes sont par ailleurs proches dans leur sens perceptif, dénotant tous deux une perception volontaire, active et attentive (ex. 65):

(65) Clérambard descend de la roulotte qu'il considère en prenant quelque peu de recul. (Marcel Aymé, I950, Frantext)

Comme regarder, et plus qu'observer, le verbe considérer se construit fréquemment avec des compléments adverbiaux précisant la manière de percevoir ou le point de vue subjectif de la perception (ex. 66, 67) :

(66) Quatre jeunes gens vêtus avec élégance le considéraient avec un sourire assez moqueur. (Julien Green, I950, Frantext)

(67) Elle le considéra d'un xil froid que la fumée de la cigarette faisait ciller. (Julien Green, I950, Frantext)

Cet aspect subjectif explique sans doute le passage fréquent vers le sens épistémique d'opinion en adoptant la structure complétive (ex. 68) et de jugement dans la structure attributive (ex. 69) :

(68) Les ouvriers ne considéreraient pas qu'ils y gagnent, et certainement se mettraient immédiatement en grève. (Simone Weil, I95 I, Frantext) 
(69) On peut considérer son livre comme des Confessions ou des Mémoires personnels [...] (Louis Bréhier, I950, Frantext)

Comme pour contempler, la perception peut aussi être purement mentale :

(70) Considérez cette chance que vous avez si peu méritée et ne soyez pas si cornichon. (Marcel Aymé, I950, Frantext)

Observer et considérer présentent tous deux une polysémie intrigante : observer combine le sens 'd'observance', historiquement le premier, avec le sens perceptif, qui l'emporte largement et reste présent dans les emplois plus cognitifs du verbe. Inversement, pour considérer, c'est le sens perceptif qui paraît actuellement déconnecté des autres, le sens évaluatif et épistémique étant aujourd'hui largement dominant.

Le Tableau 6 présente de façon synthétique les diverses structures syntaxico-sémantiques des verbes étudiés.

Tableau 6. voir, regarder, contempler, observer et considérer : structures syntaxico-sémantiques

\begin{tabular}{|c|c|c|c|c|c|c|}
\hline structures & $\begin{array}{l}\text { sens } \\
\text { structural }\end{array}$ & voir & regarder & $\begin{array}{l}\text { contem- } \\
\text { pler }\end{array}$ & observer & $\begin{array}{l}\text { consi- } \\
\text { dérer }\end{array}$ \\
\hline $\begin{array}{l}\mathrm{SN}_{1}+\mathrm{V}+ \\
\mathrm{SN}_{2} \\
\mathrm{SN}_{1}+\mathrm{V}+ \\
\mathrm{SN}_{2}+\mathrm{INF} \\
\mathrm{SN}_{1}+\mathrm{V}+ \\
\mathrm{SN}_{2}+\text { rel. } \\
\text { attrib. }\end{array}$ & $\begin{array}{l}\text { perception } \\
\text { directe }\end{array}$ & + & + & + & + & + \\
\hline $\begin{array}{l}\mathrm{SNI}+\mathrm{V}+ \\
\text { que + } \\
\text { PIND }\end{array}$ & $\begin{array}{l}\text { perception } \\
\text { indirecte/ } \\
\text { cognition/ } \\
\text { opinion }\end{array}$ & $\begin{array}{c}+ \\
\text { perception/ } \\
\text { cognition }\end{array}$ & - & - & $\begin{array}{c}\quad+ \\
\text { percep- } \\
\text { tion/co } \\
\text { gnition }\end{array}$ & $\begin{array}{c}+ \\
\text { opinion }\end{array}$ \\
\hline $\begin{array}{l}\mathrm{SNI}_{+}+\mathrm{V}+ \\
\mathrm{SN}_{2}+\mathrm{AO}\end{array}$ & évaluation & + & + & - & - & + \\
\hline
\end{tabular}

\section{Conclusion}

Cette analyse d'un certain nombre de verbes de perception illustre surtout les rapports étroits entre syntaxe et sémantique, chaque construction ou famille de constructions étant porteuse d'un sens précis, auquel 
les verbes étudiés s'adaptent selon leurs spécificités lexicales propres. Elle montre par ailleurs la complémentarité entre l'apport structural du sens, et l'apport proprement lexical de chaque lexème impliqué.

Ce champ particulier est d'autre part un lieu privilégié pour l'étude du rapport entre langue et structure conceptuelle : la diversité des modalités de la perception visuelle, la variété lexicale et la polysémie particulièrement riche des verbes impliqués, témoignent de l'importance de la vision dans notre conceptualisation du monde. Le verbe observer, de par son rôle dans le passage entre perception et cognition, en constitue un chaînon essentiel.

\section{Notes}

I. Tous les exemples cités de voir et regarder proviennent du journal Le Monde de 1997.

2. Par la perception indirecte, à la fois physique et cognitive, nous entendons la perception d'un fait par déduction à partir d'indices (ex. je vois à ta mine que tu es rentré tard hier soir) (Cf. Lemhagen I979, Willems I983).

3. Ce dérivé, ne s'appliquant qu'à ce sens spécifique du verbe observer, nous a paru la meilleure étiquette sémantique.

4. Le corpus littéraire peut présenter des emplois métaphoriques, mais qui restent dans le champ perceptif.

5. Nous utilisons le terme 'cadre sémantique' pour traduire le terme anglais 'semantic frame' (cf. Framenet)

6. Notre analyse est basée sur 2 I 5 occurrences du verbe dans la base de données Frantext (de I950 à I980)

7. Les auteurs les mieux représentés dans l'échantillon analysé sont significatifs sur ce plan (Simone Weil, Teilhard de Chardin, Camus, Simone de Beauvoir, Vladimir Jankélévitch, Jean-Paul Sartre ).

8. Notre analyse est basée sur 250 occurrences du verbe dans la base de données Frantext (de I950 à I980).

9. Je tiens à remercier Jacques François pour ses remarques pertinentes à ce sujet (communication personnelle).

\section{Références}

Baker, Collin, F. \& Josef Ruppenhofer. 2002. « FrameNet's Frames vs. Levin's Verb Classes ». In : Larson, Julie \& Mary Paster (dir.). Proceedings of the 28th Annual Meeting of the Berkeley Linguistics Society. 27-38. 
Baker, Colin F. 1999. Seeing Clearly: Frame Semantic, Psycholinguistic, and Cross-linguistic Approaches to the Semantics of the English Verb See. PhD Dissertation. UCBerkeley.

Framenet website: framenet.icsi.berkeley.edu

Hanegreefs, Hilde. 2008. Los verbos de percepción visual. Un análisis de corpus en un marco cognitivo. Thèse de doctorat non publiée sous la direction de Delbecque, Nicole \& Dominique Willems. Koninglijke Universiteit Leuven.

Lemhagen, Gunnar. 1979. La concurrence entre l'infinitif et la subordonnée complétive introduite par que en français contemporain, Stockholm: Almqvist \& Wiksell.

Miller, Philip \& Brian Lowrey. 2003. "La complémentation des verbes de perception en anglais et en français ". In : Miller, Philip \& Anne ZribiHertz (dir.). Essais sur la grammaire comparée du français et de l'anglais. Paris : Presses Universitaires de Vincennes. I3 I-I 88.

Willems, Dominique. I98I. Syntaxe, lexique et sémantique. Les constructions verbales, Publications de l'Université de Gand.

Willems, Dominique. 1983. "Regarde voir. Les verbes de perception visuelle et la complémentation verbale ». Romanica Gandensia 20. I47-I 58.

Willems, Dominique. 2000a. "Les verbes de perception et le passif ». In : Schøsler, Lene (dir.). Le passif (= Etudes Romanes 45). Copenhagen. I 7 I-I 85 .

Willems, Dominique \& Bert Defrancq. 20oob. «L'attribut de l'objet et les verbes de perception ". Langue française I 27: 6-2I.

Willems, Dominique. 2007. "Typologie des procès et régularités polysémiques ». In: Bouchard, Denis \& Ivan Evrard (dir.). Représentations du sens II, De Boeck-Duculot. I62-I77.

Willems, Dominique. 20I I. "Observer : entre regarder et voir ». In : Neveu, Franck, Peter Blumenthal \& Nicole Le Querler (dir.). Au commencement était le verbe. Syntaxe, Sémantique et Cognition. Peter Lang. Série Sciences pour la communication $97.467-478$. 